
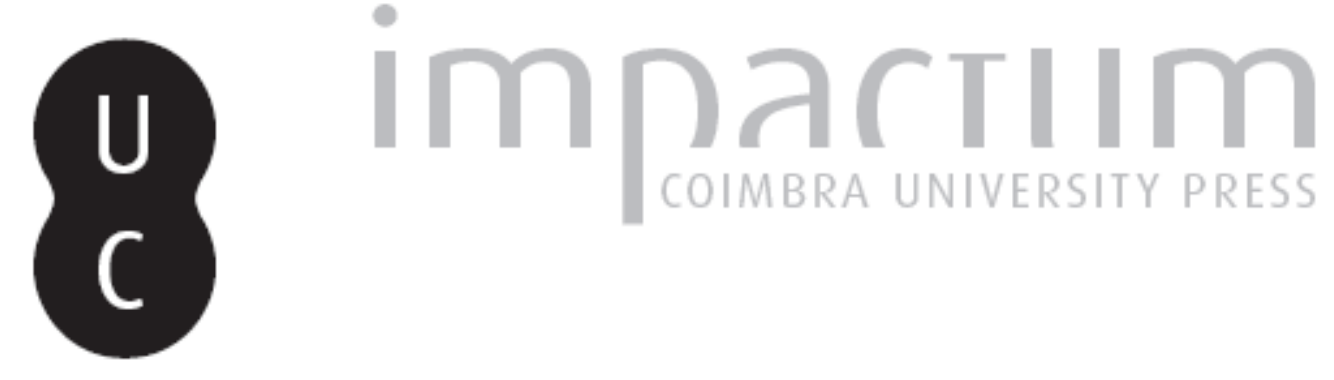

\title{
Determinantes comportamentais e obesidade entre adolescentes: tendências e variações em território Europeu
}

\author{
Autor(es): $\quad$ Lourenço, Ana; Nogueira, Helena
}

Publicado por: Imprensa da Universidade de Coimbra

URL

persistente:

URI:http://hdl.handle.net/10316.2/36893

DOI:

DOI:http://dx.doi.org/10.14195/0871-1623_33_13

Accessed : $\quad$ 26-Apr-2023 09:05:21

A navegação consulta e descarregamento dos títulos inseridos nas Bibliotecas Digitais UC Digitalis, UC Pombalina e UC Impactum, pressupõem a aceitação plena e sem reservas dos Termos e Condições de Uso destas Bibliotecas Digitais, disponíveis em https://digitalis.uc.pt/pt-pt/termos.

Conforme exposto nos referidos Termos e Condições de Uso, o descarregamento de títulos de acesso restrito requer uma licença válida de autorização devendo o utilizador aceder ao(s) documento(s) a partir de um endereço de IP da instituição detentora da supramencionada licença.

Ao utilizador é apenas permitido o descarregamento para uso pessoal, pelo que o emprego do(s) título(s) descarregado(s) para outro fim, designadamente comercial, carece de autorização do respetivo autor ou editor da obra.

Na medida em que todas as obras da UC Digitalis se encontram protegidas pelo Código do Direito de Autor e Direitos Conexos e demais legislação aplicável, toda a cópia, parcial ou total, deste documento, nos casos em que é legalmente admitida, deverá conter ou fazer-se acompanhar por este aviso.

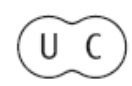




\section{Cadernos de Geografia}
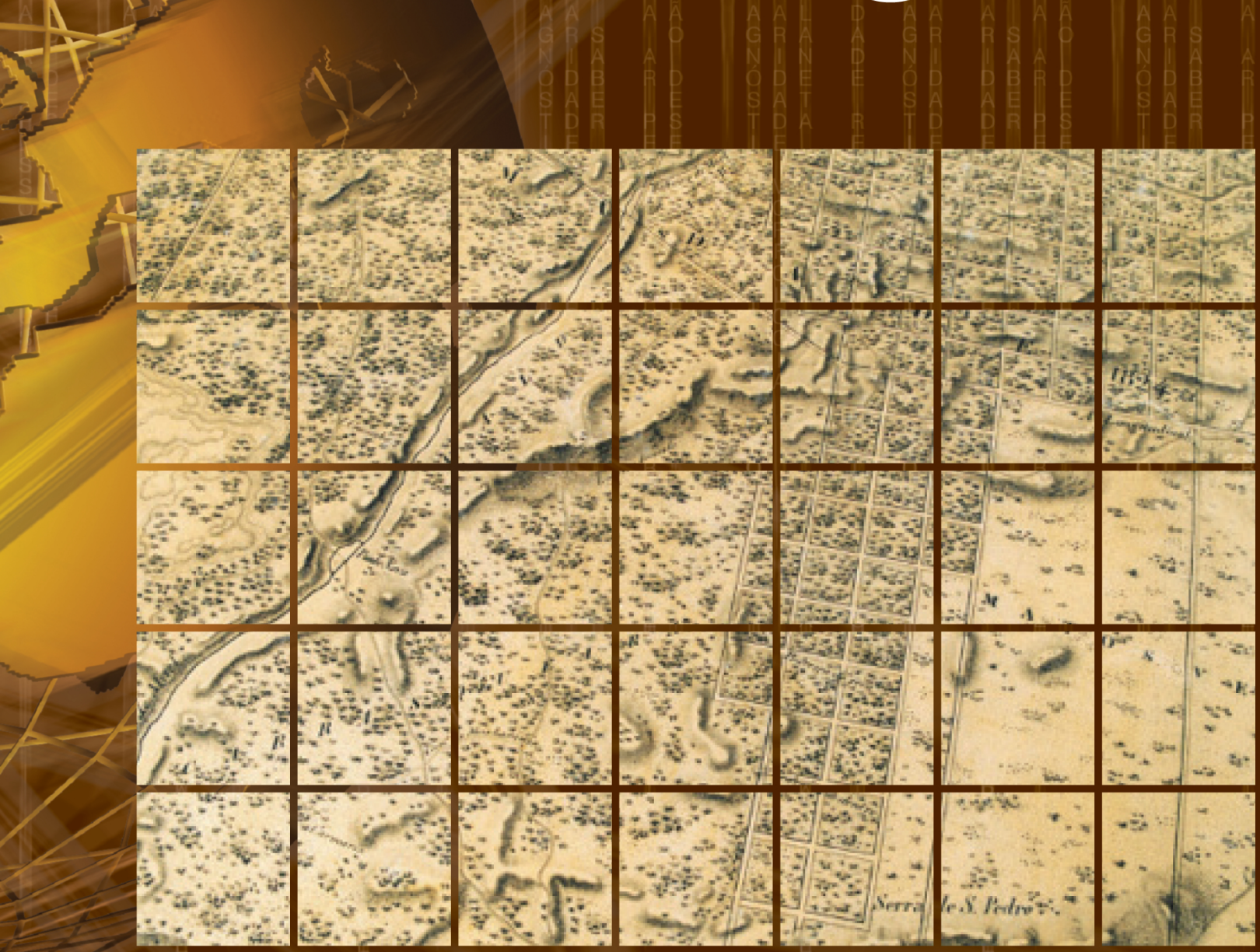

№ 33 - 2014

Imprensa da Universidade de Coimbra Faculdade de Letras | Universidade de Coimbra 


\title{
Determinantes comportamentais e obesidade entre adolescentes: tendências e variações em território Europeu Behavioral Determinants and obesity between adolescents: Trends and variations in the European territory.
}

\author{
Ana Lourenço \\ Onkoloji Hastaları Yardımlașma ve Sevgi Derneği (ONKO-SEV), Muș, Turquia. \\ claudivieira@gmail.com
}

\section{Helena Nogueira}

Departamento de Geografia, Faculdade de Letras, Universidade de Coimbra. Centro de Investigação em Antropologia e Saúde, Faculdade de Ciências da Vida, Universidade de Coimbra.

helenamarquesnogueira@hotmail.com

\section{Resumo:}

Este estudo avalia a prevalência de excesso de peso e obesidade entre jovens em idade escolar residentes em território Europeu. Apresenta e compara ainda as estimativas de consumo de vegetais, fruta e refrigerantes; níveis de atividade física; tempo gasto a ver televisão e a jogar jogos de computador e de consola. Os resultados obtidos revelam que as prevalências de excesso de peso e obesidade são superiores no sul da Europa. Sugerem também que os adolescentes do norte da Europa relatam um consumo de fruta e vegetais mais frequente e um menor consumo de refrigerantes, reportando ainda um maior nível de atividade física, enquanto os adolescentes do sul da Europa referem com mais frequência assistir televisão e jogar videojogos duas ou mais horas por dia durante a semana.

Palavras-chave: Obesidade Juvenil. Comportamentos Sedentários. Comportamentos Alimentares. Atividade Física. Europa.

\section{Abstract:}

This study assesses the prevalence of overweight and obesity among school-aged youth residing in the European territory. Also presents and compares estimates of vegetables, fruit and soft drinks consumption; levels of physical activity; time spent watching television and playing computer games or games console. The results point that the levels of overweight and obesity are highest in southern Europe. Reveal that adolescents in northern Europe are more likely to report frequent fruit and vegetable consumption, but less likely to drink soft drinks on a daily basis. Also show that adolescents in northern Europe report high levels of weekly physical activity; while adolescents in southern Europe report that they watch television and play videogames two or more hours daily on weekdays more frequently.

Keywords: Juvenile Obesity. Sedentary Behaviours. Eating Behaviours. Physical Activity. Europe. 


\section{Introdução}

As doenças crónicas e degenerativas dominam o perfil epidemiológico dos países desenvolvidos. Estas patologias, apesar de diversificadas, partilham os mesmos comportamentos de risco - inatividade física, dieta desequilibrada, tabagismo. Dois destes comportamentos estão diretamente implicados na tendência mundialmente observada de aumento de peso, revelando-se este aumento de peso, e a obesidade, como importantes contributos para o acréscimo das doenças crónicas.

A obesidade é hoje um dos maiores problemas de saúde pública da humanidade. É-o pelo seu impacto na mortalidade e na morbilidade, dado o seu papel potenciador, ou mesmo desencadeador, em várias doenças crónicas e é-o também pelas suas elevadas prevalências, tendo sido designada como a pandemia do século XXI. Trata-se de uma patologia característica de perfis epidemiológicos modernos, revelando-se como um problema multifatorial, cujo desenvolvimento tem sido atribuído a uma interação entre os genes e o ambiente (Nogueira e Lourenço, 2013). De facto, e apesar do reconhecido papel dos fatores genéticos como promotores de "suscetibilidade" a esta patologia, o aumento das prevalências entre populações geneticamente estáveis destaca o papel dos fatores ambientais e do seu efeito "obesogénico".

A adolescência representa uma fase crucial no ciclo da vida do ser humano, durante a qual muitos dos estilos de vida e comportamentos, que tendem a ser perpetuados ao longo da vida adulta, são formados e estabelecidos. Durante a adolescência, os indivíduos começam a agir de forma mais independente em relação às suas atividades de lazer e a expressar as suas preferências alimentares. É ainda na adolescência que aumenta o acesso dos indivíduos a uma variada gama de produtos alimentares, para além dos disponíveis no seu ambiente doméstico (LeECH et al., 2014). Estudos apontam que os maus hábitos alimentares e os estilos de vida sedentários, desenvolvidos na primeira infância e especialmente na adolescência, tendem a ser reforçados e mantidos na vida adulta (Telama, 2009; LAKE et al., 2006).

0 consumo atual de alimentos muito calóricos provenientes da fast-food, e os elevados níveis de sedentarismo, decorrentes do excesso de horas a ver televisão, da cada vez maior utilização de computadores e do tempo gasto com jogos eletrónicos, em detrimento de formas mais ativas de lazer, resultam num desequilíbrio entre a energia consumida e a energia despendida, desequilíbrio este que está na origem do ganho de peso e desenvolvimento de obesidade entre a população adolescente (LANDSBERG, et al, 2010).

Recentemente, foi revelado que a prevalência de obesidade na vida adulta está fortemente relacionada com a obesidade que se estabelece no final da infância e na adolescência ${ }^{1}$. Foi demons-

\footnotetext{
Vários autores apontam que cerca de $80 \%$ dos adolescentes obesos tornam-se adultos obesos e cerca de um terço dos adultos obesos já o eram em criança, acarretando implicações na qualidade de vida, na morbilidade e na mortalidade (Guo et al., 1999).
}

trado, por outro lado, que os adolescentes obesos tendem a ser adultos mais obesos do aqueles que se tornam obesos apenas na idade adulta (Guo et al., 1998). Desta forma, o motivo de preocupação é duplo. Em primeiro lugar, porque vários estudos mostram que a obesidade pode manter-se desde a infância até à vida adulta. E em segundo lugar, porque a obesidade na adolescência está diretamente associada com o aumento da morbilidade e mortalidade na vida adulta, independentemente do peso corporal.

\section{Dados e Métodos}

\section{Amostra}

No presente estudo utilizaram-se os resultados do inquérito "Health Behaviour in School-aged Children" (HBSC) de 2009-2010. O HBSC, um inquérito transnacional elaborado com colaboração da Organização Mundial de Saúde, procura identificar os comportamentos de saúde dos adolescentes em idade escolar, os seus estilos de vida e os seus contextos sociais em 43 países, incluindo Portugal. Dos 43 países que participaram no estudo, apenas 39 alcançaram as diretrizes exigidas para a publicação dos seus resultados no HBSC de 2009-2010² (Currie, et al., 2012: 2). Para garantir amostras nacionalmente representativas da população adolescente residente em cada país analisado (com 11, 13 e 15 anos), o HBSC recomendou a seleção de uma amostra de 1500 adolescentes em cada grupo de idade, tamanho que não era previsto alcançar na Islândia e Gronelândia, dado o quantitativo demográfico destes países. Refira-se ainda que vários países optaram por utilizar amostras superiores às recomendadas, a fim de aumentar a precisão das estimativas (Quadro I). Em todos os países foram excluídos os adolescentes que não frequentavam a escola, ou que frequentavam es colas para crianças e jovens com necessidades de apoio especial (CURRIE, et al., 2012: 222). A recolha dos dados foi realizada entre Setembro de 2009 e Julho de 2010, através de questionários administrados nas salas de aula, sendo o seu preenchimento anónimo e voluntário. Os questionários foram aplicados por investigadores ou professores, usando um protocolo padrão fornecido pelo grupo de investigadores que se encontrava a realizar trabatho de campo. O preenchimento dos questionários foi efetuado pelos adolescentes, após obtenção de consentimento ético.

\footnotetext{
O não envio dos dados dentro dos prazos estabelecidos e a incapacidade para garantir o financiamento do estudo, foram as principais razões apontadas para a exclusão de 4 dos 43 países participantes no HBSC de 2009-2010.
} 
Quadro I

Total de participantes por país, segundo o género e idade

\begin{tabular}{|c|c|c|c|c|c|c|}
\hline \multirow{2}{*}{ País } & \multicolumn{2}{|c|}{ Género } & \multicolumn{3}{|c|}{ Idades } & \multirow{2}{*}{ Total } \\
\hline & Rapazes & Raparigas & 11 anos & 13 anos & 15 anos & \\
\hline Arménia & 1343 & 1490 & 889 & 1029 & 915 & 2833 \\
\hline \begin{tabular}{|l|} 
Áustria \\
\end{tabular} & 2456 & 2547 & 1457 & 1726 & 1820 & 5003 \\
\hline \begin{tabular}{|l|} 
Bélgica \\
(Flamenga)
\end{tabular} & 2086 & 2094 & 1501 & 1453 & 1226 & 4180 \\
\hline $\begin{array}{l}\text { Bélgica } \\
\text { (Francesa) }\end{array}$ & 1985 & 2027 & 1275 & 1396 & 1341 & 4012 \\
\hline Canadá & 7711 & 7999 & 4490 & 5779 & 5441 & 15710 \\
\hline Croácia & 3012 & 3240 & 1879 & 1949 & 2424 & 6252 \\
\hline $\begin{array}{l}\text { República } \\
\text { Checa }\end{array}$ & 2135 & 2269 & 1426 & 1456 & 1522 & 4404 \\
\hline Dinamarca & 1914 & 2132 & 1558 & 1262 & 1226 & 4046 \\
\hline Inglaterra & 1522 & 1981 & 1185 & 1200 & 1118 & 3503 \\
\hline Estónia & 2022 & 2202 & 1416 & 1410 & 1398 & 4224 \\
\hline Finlândia & 3179 & 3428 & 2345 & 2152 & 2110 & 6607 \\
\hline França & 3030 & 2990 & 2042 & 2072 & 1906 & 6020 \\
\hline Alemanha & 2406 & 2549 & 1687 & 1628 & 1640 & 4955 \\
\hline Grécia & 2380 & 2519 & 1639 & 1612 & 1648 & 4899 \\
\hline Gronelândia & 586 & 619 & 384 & 424 & 397 & 1205 \\
\hline Hungria & 2257 & 2530 & 1473 & 1581 & 1733 & 4787 \\
\hline Islândia ${ }^{3}$ & 5569 & 5480 & 3623 & 3746 & 3680 & 11049 \\
\hline Irlanda & 2522 & 2202 & 1148 & 1881 & 1695 & 4724 \\
\hline Itália & 2408 & 2403 & 1585 & 1680 & 1546 & 4811 \\
\hline \begin{tabular}{|l|} 
Letónia \\
\end{tabular} & 2054 & 2210 & 1492 & 1397 & 1375 & 4264 \\
\hline Lituânia & 2740 & 2583 & 1811 & 1720 & 1792 & 5323 \\
\hline \begin{tabular}{|l|} 
Luxemburgo \\
\end{tabular} & 2044 & 2028 & 1079 & 1611 & 1382 & 4072 \\
\hline Holanda & 2219 & 2301 & 1483 & 1580 & 1457 & 4520 \\
\hline Noruega & 2171 & 2167 & 1679 & 1320 & 1339 & 4338 \\
\hline Polónia & 2065 & 2176 & 1395 & 1436 & 1410 & 4241 \\
\hline Portugal & 1878 & 2158 & 1183 & 1300 & 1553 & 4036 \\
\hline Roménia & 2647 & 2705 & 1624 & 1726 & 2002 & 5352 \\
\hline \begin{tabular}{|l|} 
Federação \\
Russa $^{4}$
\end{tabular} & 2576 & 2598 & 2052 & 1275 & 1847 & 5174 \\
\hline Escócia & 3319 & 3419 & 2055 & 2116 & 2567 & 6738 \\
\hline Eslováquia & 2561 & 2720 & 1427 & 1940 & 1914 & 5281 \\
\hline Eslovénia & 2761 & 2668 & 1803 & 1811 & 1815 & 5429 \\
\hline Espanha & 2466 & 2574 & 1257 & 1780 & 2003 & 5040 \\
\hline Suécia & 3312 & 3333 & 2264 & 2291 & 2090 & 6645 \\
\hline Suíça & 3320 & 3291 & 1843 & 2522 & 2246 & 6611 \\
\hline Macedónia & 1952 & 1945 & 1079 & 1282 & 1536 & 3897 \\
\hline Turquia & 2652 & 2922 & 1902 & 1912 & 1760 & 5574 \\
\hline Ucrânia & 2809 & 3081 & 2131 & 1862 & 1897 & 5890 \\
\hline $\begin{array}{l}\text { Estados Unidos } \\
\text { da América }\end{array}$ & 3260 & 3014 & 1903 & 2479 & 1892 & 6274 \\
\hline País de Gales & 2746 & 2665 & 1885 & 1889 & 1637 & 5411 \\
\hline Total & 102075 & 105259 & 66349 & 70685 & 70300 & 207334 \\
\hline
\end{tabular}

Fonte: Elaborado a partir de Currie, et al., pp. 224 (2012)

Do questionário, que engloba um vasto conjunto de questões sobre comportamentos e estilos de vida na adolescência, foram selecionadas para o presente estudo as questões que permitem caracterizar o estado nutricional, níveis de atividade física, ocupação dos tempos livres e hábitos alimentares dos adolescentes residentes em território Europeu.

\section{Medidas Antropométricas}

Cada adolescente indicou o seu peso corporal (sem roupa) e a sua altura (sem sapatos) nas unidades apropriadas de cada país (centímetros ou polegadas, libras ou quilogramas). Os valores em libras e polegadas foram recodificados em quilogramas e centímetros, respetivamente. $O$ Índice de Massa Corporal (IMC)

\footnotetext{
3 Na Islândia considerou-se adequada a realização de um levamento censitário, devido à pequena proporção de jovens residentes neste país (CURRIE, et al., 2012, pp. 222).

${ }^{4} \mathrm{Na}$ Federação Russa, a base de amostragem cobre apenas algumas regiões e não o território nacional total (CURRIE, et al., 2012, pp. 222).
}

foi calculado como o quociente entre o peso, expresso em quilogramas, e o quadrado da estatura, expresso em metros $\left(I M C=\mathrm{Kg} / \mathrm{m}^{2}\right)$. 0 excesso de peso e a obesidade foram definidos utilizando os pontos de corte do IMC, específicos para o sexo e idade, publicados pelo International Obesity Task Force (IOTF).

\section{Comportamentos Alimentares}

Os adolescentes foram interrogados sobre quantas vezes, numa semana típica, consumiam fruta, vegetais e refrigerantes ${ }^{5}$. As opções de resposta eram: "nunca"; "menos de uma vez por semana"; "uma vez por semana"; "2-4 dias por semana"; " 5-6 dias por semana"; "uma vez, todos os dias"; e "mais do que uma vez, todos os dias". Na presente análise irão ser apenas apresentados os valores relativos aos adolescentes que reportaram beber refrigerantes e comer fruta e vegetais "uma vez, todos os dias" ou "mais do que uma vez, todos os dias".

\section{Comportamentos Sedentários}

Os adolescentes foram questionados sobre quantas horas por dia, numa semana típica, viam televisão (incluindo vídeos e DVDs) e jogavam videojogos (jogos de computador ou de consola) nos seus tempos livres, durante os dias de semana e fim-de-semana, separadamente. Os resultados apresentados referem-se à proporção de adolescentes que relatam ver televisão ou jogar jogos de computador e de consola por duas ou mais horas todos os dias da semana.

\section{Níveis de Atividade Física}

Após definição de atividade física ${ }^{6}$ e apresentação de alguns exemplos de tais atividades, os jovens foram questionados sobre o número de horas que, por semana, eram fisicamente ativos nos seus tempos livres fora do horário escolar. São aqui somente apresentados os dados referentes à proporção de adolescentes que participa em atividades físicas vigorosas duas ou mais horas por semana.

\section{Resultados}

Em 2009-2010, as taxas de prevalência de excesso de peso e obesidade eram superiores nos países da Europa Mediterrânica, onde mais de 16\% dos adolescentes apresentavam excesso de peso e obesidade. Observou-se, por outro lado, que na maioria dos países estudados as prevalências de excesso de peso e obesidade eram inferiores entre os indivíduos do sexo feminino (Figura 1), independentemente da idade.

\footnotetext{
5 Foram considerados como refrigerantes as Coca-colas e as bebidas açucaradas.

6 Atividade física foi definida como "qualquer atividade que aumenta a frequência cardíaca e faz perder o fôlego algumas vezes".
} 
Em Portugal, o excesso de peso e obesidade afetava mais de $18 \%$ da população adolescente, observando-se taxas de prevalência superiores entre os adolescentes do sexo masculino e os mais jovens (Figura 2).

Segundo os resultados do Health Behaviour in School-aged Children (HBSC), o consumo de vegetais é percentualmente mais baixo entre os adolescentes residentes nos países da Europa Mediterrânica e do Báltico, onde menos de $31 \%$ dos adolescentes referem comer uma ou mais porções diárias de vegetais. Mostram, por outro lado, que os adolescentes residentes nos países da Europa do Norte e de Leste são os que apresentam taxas de consumo de fruta mais baixas (menos de $32 \%$ dos indivíduos residentes na Suécia, Finlândia, Estónia, Letónia, Lituânia e Polónia afirmaram não comer fruta todos os dias). Revelam, ainda, que no período em estudo o consumo de vegetais e fruta era inferior entre os indivíduos do sexo masculino (Figura 3 e 4).

Constatou-se, por outro lado, que os adolescentes residentes em países da Europa de Leste e Ocidental reportam os valores mais elevados de consumo excessivo de coca-cola ou outro tipo de refrigerante, com mais de $23 \%$ dos adolescentes a assinalar a ingestão diária de uma ou mais doses deste tipo de bebidas. Quando se observa o padrão de consumo de refrigerantes desagregado segundo o género, verifica-se que são os adolescentes do sexo masculino que relatam consumos mais elevados de coca-cola e de outro tipo de bebidas açucaradas (Figura 5 ), em todos os grupos de idade.

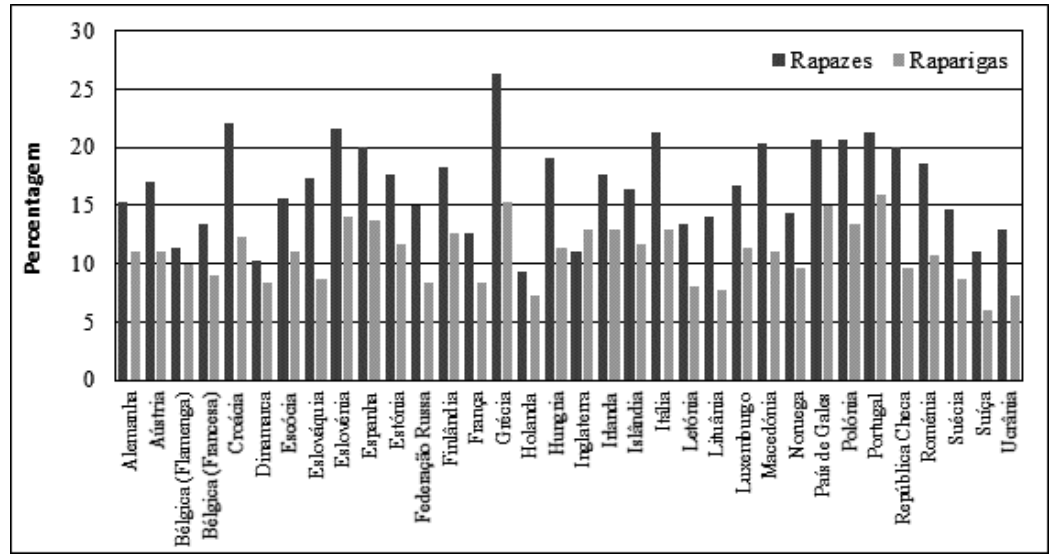

Figura 1

Percentagem de adolescentes residentes na Europa, segundo o género, que apresentou excesso de peso ou obesidade, em 2009-2010

Fonte: Elaborado a partir de CURRIE, et al., (2012)

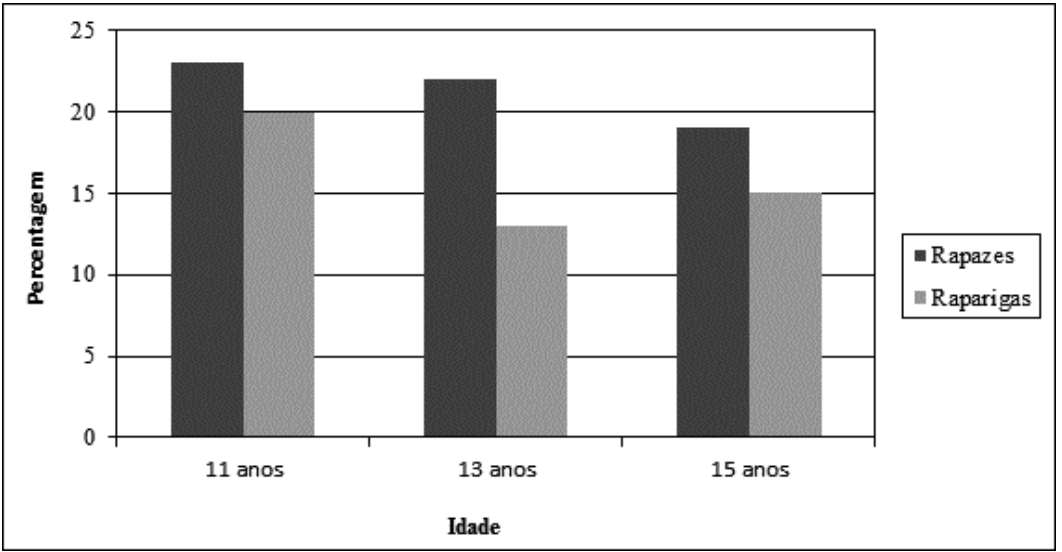

Figura 2

População inquirida em Portugal que apresentou excesso de peso ou obesidade, segundo o género e a idade, em 2009-2010

Fonte: Elaborado a partir de Currie, et al., (2012) 


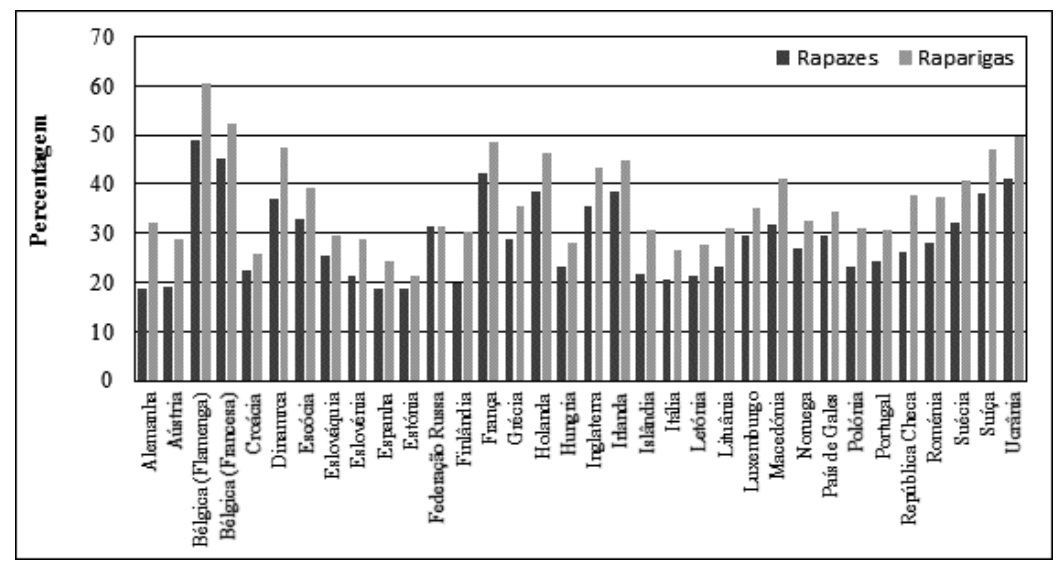

Figura 3

Percentagem de adolescentes residentes na Europa, segundo o género, que referiu ingerir uma ou mais porções de vegetais todos os dias, em 2009-2010

Fonte: Elaborado a partir de CURRIE, et al., (2012)

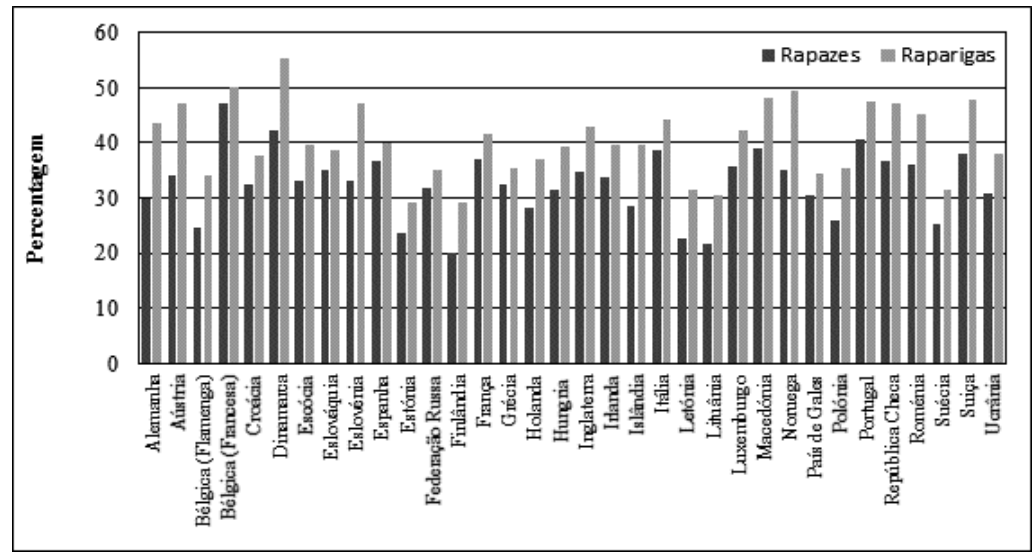

Figura 4

Percentagem de adolescentes residentes na Europa, segundo o género, que referiu comer uma ou mais porções de fruta todos os dias, em 2009-2010

Fonte: Elaborado a partir de CURRIE, et al., (2012)

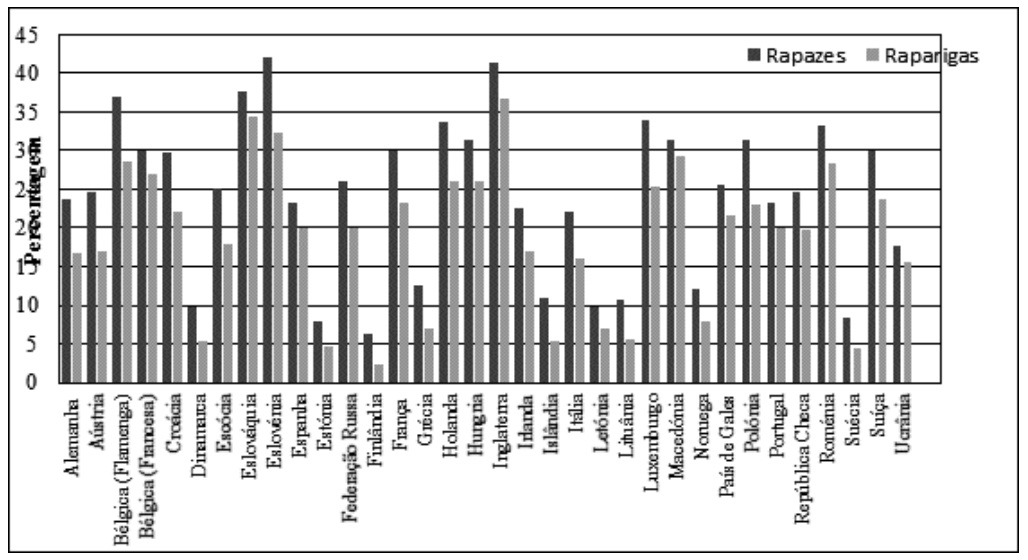

Figura 5

Percentagem de adolescentes residentes na Europa, segundo o género, que referiu beber uma ou mais doses de refrigerante todos os dias, em 2009-2010

Fonte: Elaborado a partir de CURRIE, et al., (2012) 
Para Portugal, os resultados do HBSC revelam que apenas $27,5 \%$ dos adolescentes ingeriam vegetais diariamente, evidenciando um maior consumo nas raparigas e nos adolescentes mais jovens (Figura 6). Quanto ao consumo de fruta, 43,8\% dos adolescentes portugueses referiam ingerir uma ou mais porções diárias, observando-se maiores valores de consumo diário para as raparigas e adolescentes mais novos (Figura 7). Além disso, verifica-se que $21,1 \%$ dos adolescentes portugueses declaravam beber refrigerante pelo menos uma vez por dia, sendo a frequência na população masculina superior à observada na população feminina (Figura 8). Constatou-se também que o consumo deste tipo de produtos é menor entre os adolescentes mais novos.

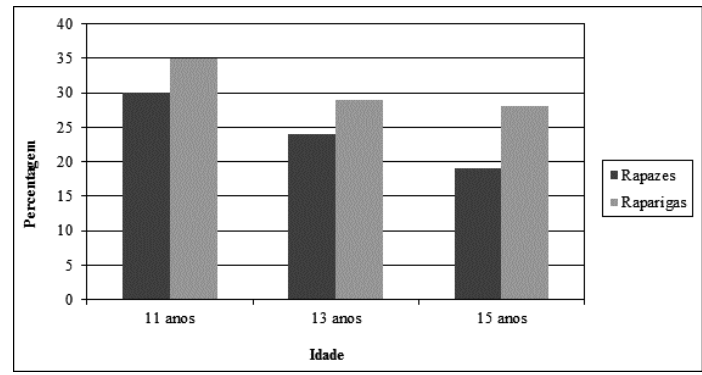

Figura 6

População inquirida em Portugal que consumia uma ou mais porções diárias de vegetais, segundo o género e a idade, em 2009-2010

Fonte: Elaborado a partir de CURRIE, et al., (2012

Por outro lado, os resultados do HBSC demonstram que os adolescentes residentes nos países da Europa do Norte são fisicamente mais ativos: mais de $63 \%$ dos adolescentes Holandeses, Dinamarqueses e Noruegueses declaram praticar atividade física, pelo menos duas horas por semana, mais frequentemente. Revelam, ainda, que é a população masculina que referencia uma maior participação em

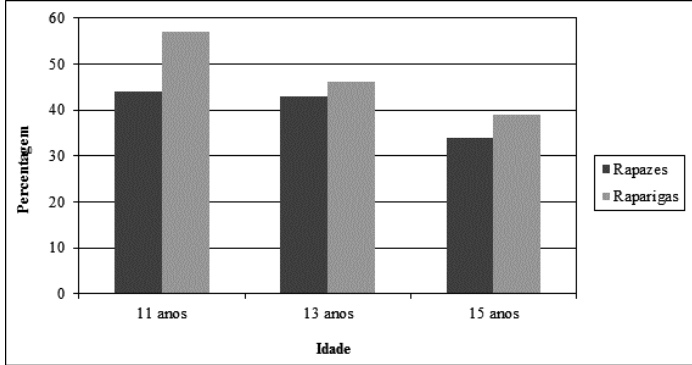

Figura 7

População inquirida em Portugal que consumia uma ou mais porções diárias de fruta, segundo o género e a idade, em 2009-2010

Fonte: Elaborado a partir de CURRIE, et al., (2012)

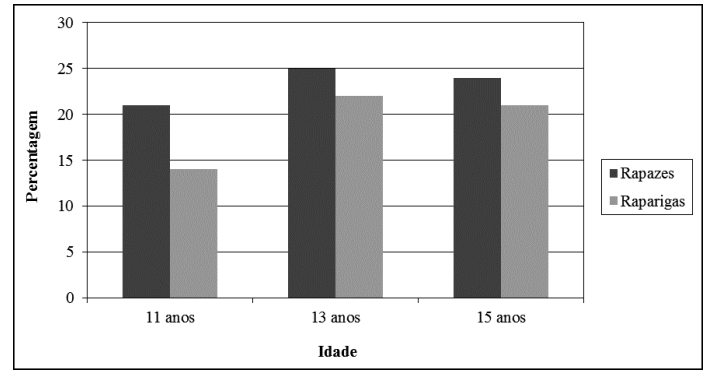

Figura 8

População inquirida em Portugal que consumia uma ou mais doses diárias de refrigerante, segundo o género e a idade, em 2009-2010 Fonte: Elaborado a partir de CURRIE, et al., (2012)

atividades físicas de intensidade vigorosa (Figura 9), em quase todos os grupos de idade estudados. Em Portugal, segundo a mesma fonte, $38,3 \%$ dos adolescentes portugueses referiam praticar exercício físico, de intensidade vigorosa, pelo menos duas horas por semana. Os rapazes e os adolescentes mais velhos referem ser mais ativos do que as raparigas e os adolescentes mais novos (Figura 10).

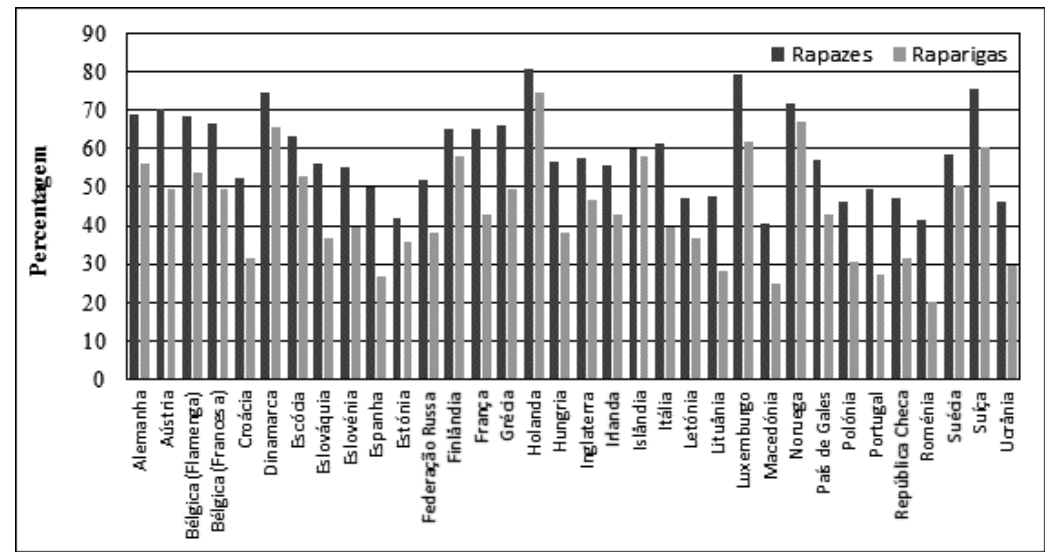

Figura 9

Percentagem de população adolescente que referiu praticar duas ou mais horas por semana de atividades físicas de intensidade vigorosa, em 2009-2010

Fonte: Elaborado a partir de CURRIE, et al., (2012) 


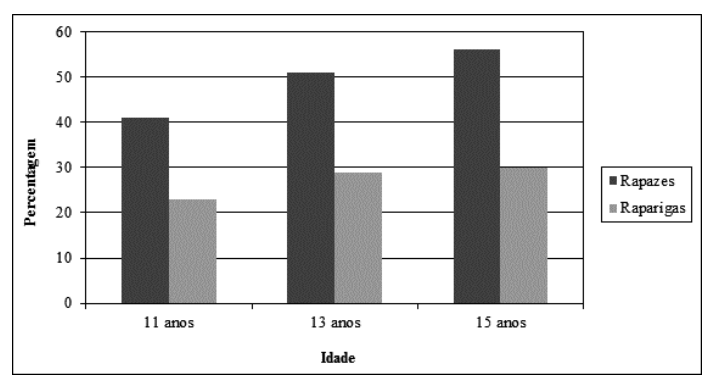

\section{Figura 10}

População inquirida em Portugal que praticava semanalmente atividades físicas de intensidade vigorosa por duas ou mais horas, segundo o género e a idade, em 2009-2010

Fonte: Elaborado a partir de CURRIE, et al., (2012)

Quando se analisa a variação geográfica na utilização de computador e consola de jogos, verifica-se que os adolescentes residentes nos países da Europa de Leste reportam uma maior utilização deste tipo de equipamentos, com mais de $35 \%$ destes adolescentes a assinalar um maior número de horas a jogar jogos de consola e computador. Todavia, em termos de visionamento televisivo, verificam- se valores mais elevados entre os adolescentes residentes em países da Europa de Leste, Mediterrânica (Grécia) e Balcãs (Croácia), onde mais de 67\% dos adolescentes referem ver duas ou mais horas diárias de televisão. Observando as figuras 11 e 12 verifica-se, ainda, uma tendência para maiores visionamentos televisivos e uso de jogos eletrónicos nos adolescentes do sexo masculino, em todos os grupos de idades, registando-se algumas (poucas) exceções, a ocorrerem sobretudo em países da Europa de Leste, Balcãs e Báltico.

Para Portugal, o presente estudo revela que $35 \%$ dos adolescentes afirmaram jogar computador ou consola durante, pelo menos, uma hora por dia, sendo este comportamento mais frequente nos adolescentes mais velhos e do sexo masculino (Figura 13). Revela, ainda, que 66,3\% dos adolescentes portugueses declaravam ver televisão duas ou mais horas por dia, sendo este comportamento menos frequente nos adolescentes mais novos (Figura 14).

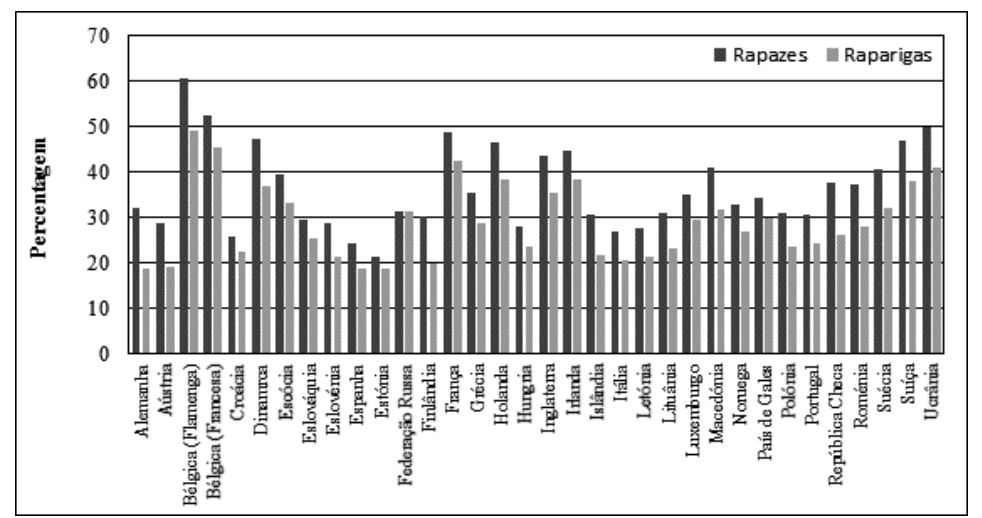

Figura 11

Percentagem de adolescentes residentes na Europa, segundo o género, que referiu jogar videojogos duas ou mais horas todos os dias, em 2009-2010 Fonte: Elaborado a partir de CURRIE, et al., (2012)

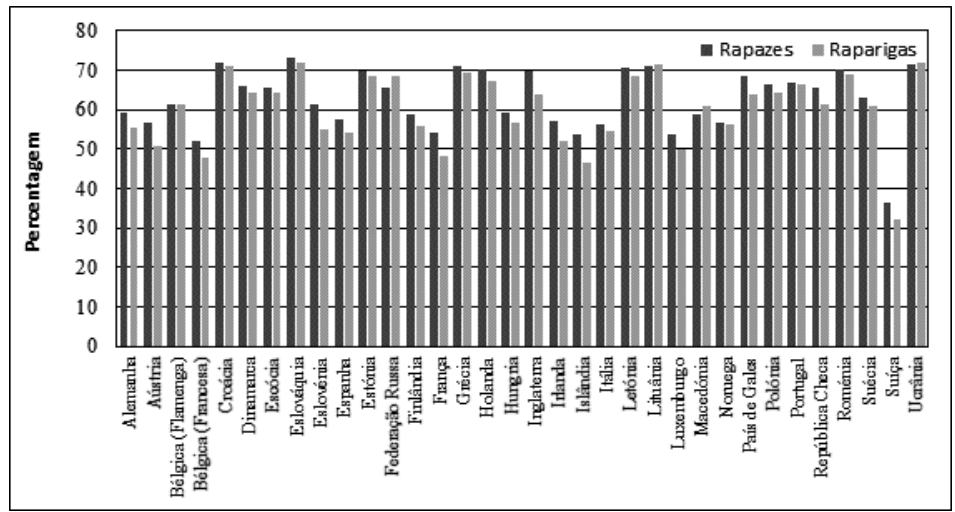

Figura 12

Percentagem de adolescentes residentes na Europa, segundo o género, que referiu ver televisão duas ou mais horas todos os dias, em 2009-2010

Fonte: Elaborado a partir de CURRIE, et al., (2012) 


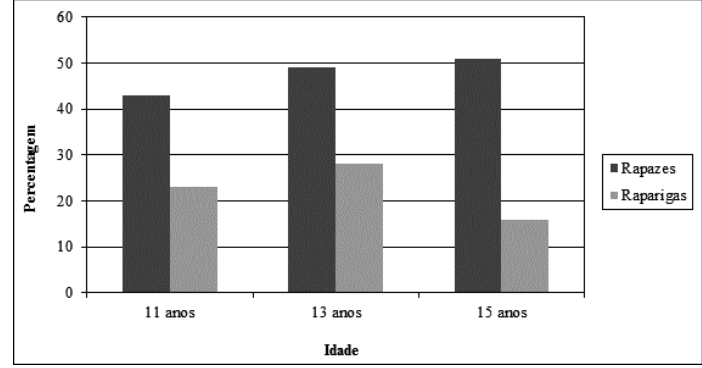

Figura 13

População inquirida em Portugal que jogava videojogos duas ou mais horas por dia, segundo o género e a idade, em 2009-2010 Fonte: Elaborado a partir de CURRIE, et al., (2012)

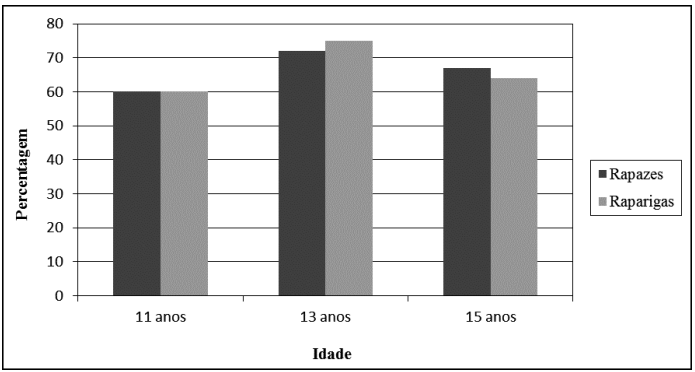

Figura 14

População inquirida em Portugal que via duas ou mais horas de televisão por dia, segundo o género e a idade, em 2009-2010

Fonte: Elaborado a partir de CURRIE, et al., (2012)

\section{Discussão e Conclusões}

A análise efetuada revelou que a prevalência de excesso de peso e obesidade juvenil, em 2009-2010, era superior nos países da Europa Mediterrânea, nomeadamente Portugal, Espanha, Itália e Grécia. Revelou também que na maioria dos países examinados o excesso de peso e obesidade afetava mais frequentemente os adolescentes do sexo masculino. Estes resultados corroboram estudos anteriores, que mostram prevalências de excesso de peso e obesidade superiores nos países do sul da Europa (LoBSTEIN e Frelut, 2003) e no sexo masculino ${ }^{7}$ (Krassas et al., 2001). Vários estudos realizados em Portugal Continental revelam também que o excesso de peso e obesidade afetam mais os adolescentes do sexo masculino, comparativamente aos do sexo feminino. Refira-se, a título de exemplo, o trabalho de AMARAL, et al. (2007), abrangendo adolescentes dos 12 aos 18 anos residentes no distrito de Viseu, onde os autores evidenciaram prevalências de excesso de peso de $16 \%$ no sexo masculino e $11,6 \%$ no sexo feminino. Os mesmos autores apontaram ainda taxas

\footnotetext{
Contrariamente ao descrito no HBSC de 2009-2010, alguns estudos apontam que na Croácia (PéIN et al., 2013); Noruega (Júlíusson et al., 2010); Holanda (SCHÖNBECK et al., 2001); Irlanda (WHELTON et al., 2007); Lituânia (TuTKUVIENE, et al., 2007) e Escócia (CHINn et al., 2001), os adolescentes do sexo feminino apresentam taxas de prevalência de excesso de peso e obesidade ligeiramente superiores aos dos adolescentes do sexo masculino.
}

de prevalência de obesidade de $4,2 \%$, no sexo mas culino e de $2,8 \%$, no sexo feminino.

A análise dos resultados do HBSC revelou que, tendencialmente, são os adolescentes do sexo masculino que relatam hábitos alimentares menos saudáveis, nomeadamente o menor consumo de vegetais e fruta, e maior ingestão de refrigerantes. Conclui-se ainda que, embora os adolescentes do sexo masculino declarem ser fisicamente mais ativos, estes tendem, mais frequentemente, a envolver-se em formas de lazer consideradas sedentárias. Estes resultados são semelhantes aos encontrados em outros estudos epidemiológicos, inclusive realizados em Portugal, que comprovam que as crianças e os adolescentes do sexo masculino são menos propensos a adotar estilos de vida saudáveis (PADEZ et al., 2004). As disparidades territoriais e de género nas prevalências de excesso de peso e obesidade observadas poderão refletir, quer os efeitos dos fatores ambientais e culturais, quer diferenças nos níveis de sedentarismo e nos padrões alimentares adotados. Note-se que alguns estudos, realizados entre crianças e adolescentes, sublinham a relação entre a incidência de obesidade e o consumo de refrigerantes. Refira-se, por exemplo, o estudo desenvolvido por Ludwig et al. (2001), onde os autores concluíram que a probabilidade das crianças e pré-adolescentes apresentarem obesidade aumentava 1,6 vezes por cada dose adicional de refrigerante consumida diariamente. De modo semelhante, Giammattel et al. (2003) revelaram que a quantidade diária de refrigerantes consumidos está significativamente relacionada com o IMC e com a percentagem de gordura corporal. Segundo os mesmos autores, os adolescentes que consomem 3 ou mais refrigerantes por dia são mais propensos a possuir uma maior percentagem de gordura corporal e um IMC igual ou superior a 85 (excesso de peso ou obesidade). Os autores referem ainda que o consumo de refrigerantes dietéticos está significativamente associado com o IMC e ganho de gordura corporal, o que não é consistente com outras investigações prévias.

Relativamente aos comportamentos sedentários, vários autores têm mostrado que o aumento nas prevalências de obesidade juvenil pode ser atribuído a baixos níveis de atividade física associados a um estilo de vida sedentário. Nos Estados Unidos da América, GoRTMAKer et al. (1996), por exemplo, ao estudarem a relação entre os valores de prevalência de obesidade e o número de horas que os jovens passavam a ver televisão, verificaram que as taxas de prevalência de obesidade eram menores entre os adolescentes que viam menos de 2 horas de televisão por dia e maiores entre aqueles que viam mais de 5 horas de televisão por dia, mesmo quando controlado o efeito do excesso de peso inicial, excesso de peso maternal, raça/etnia e estatuto socioeconómico. Segundo os autores, mais de $60 \%$ da incidência de excesso de peso podia estar associada ao excessivo consumo televisivo. Num estudo longitudinal com crianças americanas, DIETz et al. (1995) revelaram que o consumo televisivo era um fator determinante 
para o desenvolvimento de excesso de peso e obesidade, mesmo quando controlado um conjunto de fatores previamente reconhecidos por afetarem o desenvolvimento de obesidade infantil. Verificaram também um aumento das taxas de prevalência de excesso de peso e obesidade de 1,2\% para $2,9 \%$ e de $1,4 \%$ para $1,6 \%$ por cada hora adicional de televisão visionada por dia, respetivamente. Uma relação semelhante, entre visionamento televisivo e obesidade, foi observada em crianças e adolescentes com idades compreendidas entre os 9 e 16 anos residentes no México (Hernandez, et al., 1999). Na Bélgica, Guillaume et al. (1997), demostraram que esta relação era apenas significativa para os rapazes, sugerindo que os jovens do sexo masculino são mais sensíveis a alguns fatores de risco para o desenvolvimento desta doença.

Alguns estudos sugerem ainda a existência de uma associação positiva entre o uso de jogos eletrónicos e a incidência de obesidade. Refira-se, como exemplo, o trabalho de VANDEWATER et al. (2004), onde os autores destacam a existência de uma relação linear positiva entre o uso de jogos eletrónicos e o IMC, para uma amostra nacionalmente representativa de crianças entre os 1 e os 12 anos, residentes nos Estados Unidos da América. De forma similar, TREMBLAY e WiLliams (2003), após terem controlado alguns fatores confundentes como o sexo, a idade e o estatuto socioeconómico do agregado familiar, verificaram que as crianças canadianas com idades compreendidas entre os 7 e os 11 anos apresentam maior probabilidade de padecer de excesso de peso $(17 \%)$ e obesidade $(10 \%)$ quando jogam videojogos por períodos superiores a 2 horas diárias.

As diferenças de género observadas podem indicar, como foi anteriormente sugerido, uma maior sensibilidade dos indivíduos do sexo masculino às influências ambientais, ou ainda uma menor eficiência das intervenções preventivas para os indivíduos deste género. É importante ressaltar que as raparigas, dada a sua tendência de ganharem gordura corporal durante a puberdade, contrariando os estereótipos de beleza feminina, são frequentemente encorajadas pelos pais a controlarem o seu peso, adotando práticas dietéticas com mais facilidade. Ou seja, é provável que as diferenças de género resultem de um conjunto interativo de diferenças biológicas, sociais e culturais

Por último, os resultados deste estudo enfatizam a necessidade de estimular a prática regular de exercício físico entre as crianças e adolescentes, o que poderá passar por campanhas de sensibilização que sublinhem as vantagens de desenvolver estilos de vida ativos e saudáveis e pela oferta de modalidades desportivas que respondam às preferências destes grupos populacionais. Revelam ainda que é necessário melhorar a dieta dos adolescentes, sobretudo a do sexo masculino, sensibilizando-os para as vantagens de uma alimentação saudável, o que passa por intervenções precoces e atempadas que evitem a aquisição de maus hábitos alimentares que, como foi observado, se vão tornando mais frequentes com o aumento da idade.

\section{Referências Bibliográficas}

Berg, I. M. C.; Simonsson, B.; Brantefor, B. e Ringgvist, I. (2001) "Prevalence of overweight and obesity in children and adolescents in a county in Sweden". Acta Paediatrica, 90(6), pp. 671-676.

Berkey, C. S.; Rockett, H. R.; Field, A. E.; Gillman, M. W. e Colditz, G. A. (2004) - "Sugar-added beverages and adolescent weight change”. Obesity Research, 12(5), pp. 778-788.

CHINN, S. e RonA, R. J. (2001) - "Prevalence and trends in overweight in three cross-sectional studies in British children, 1974-1994". British Medical Journal, 322, pp. 24-26.

Currie, C.; Zanotti, C.; Morgan, A.; Currie, D.; de Looze, M.; Roberts, C.; Samdal, O.; Smith, O. e Barnekow, V. (2012) - Social determinants of health and well being among young people. Health Behaviour in School-Aged Children (H.B.S.C.) study: International report from the 2009/2010. Organização Mundial de Saúde, Copenhaga.

Dietz, W. H. e Gortmaker, S. L. (1985) - "Do we fatten our children at the TV set? Obesity and television viewing in children and adolescents". Pediatrics, 75(5), pp. 807-8012.

Еквцом, O.; Oddsson, K. е Еквцом, В. (2004) - "Prevalence and regional differences in overweight in 2001 and trends in BMI distribuition in Swedish children from 1987 to 2001". Scandinavian Journal of Public Health, 32(4), pp. 257-263.

Emandi, A.; Puiu, M.; Gafencu, M. e Pienar, C. (2013) - "Arterial hypertension in school aged children in western Romania”. Cardiology in the Young, 23(2), pp. 189-196.

Freisling, H. e Elmadfa, I. (2007) - "Prevalence of overweight and obesity among Austrian children (3-15 y): using three different sets of reference values for body mass index (BMI)". Nutrition, 31(1), pp. 6-11.

Gazina, D. N. e Gurevich, K. G. (2011) - "Obesity in Moscow school-age students: Prevalence, Age- and Gender Related Features". Food and Public Health, 1(1), pp. 1-5.

Giammattel, J.; Blix, G.; Marshak, H. H.; Wollitzer, A. O. e Pettitt, D. J. (2003) - "Television watching and soft drink consumption: associations with obesity in 11 to 13 year-old schoolchildren". Archives of Pediatrics and Adolescent Medicine, 157(9), pp. 882-886.

Gortmaker, S. L.; Must, A.; Sobol, A. M.; Peterson, K.; Colditz, S. A. e Dietz, W. H. (1996) - "Television viewing as a cause of increasing obesity among children in the United States, 1986-1990". Archives of Pediatrics and Adolescent Medicine, 150(4), pp. 356-362.

Guillaume, M.; Lapidus, L.; Bjorntorp, P. e Lambert, A. (1997) "Physical activity, obesity and cardiovascular risk factors in Children: The Belgian Luxembourg Child Study II". Obesity Research, 5(6), pp. 549-556. 
Guo, S. S. e Chumlea, W. C. (1999) - "Tracking of body mass index in children in relation to overweight in adulthood". American Journal of Clinical Nutrition, 70(1), pp. 145-148.

Hernández, B.; Gortmaker, S. L.; Colditz, G.A.; Peterson, K. E.; Laird, N. M. e Parra-Caberra, S. (1999) - "Association of obesity with physical activity, television programs and other forms of video viewing among children in Mexico City". International Journal of Obesity and Related Metabolic Disorders, 23(8), pp. 845-854.

JodkowsKa, M.; OBlaCinSKA, A. e TABAK, I. (2010) - "Overweight and obesity among adolescents in Poland: gender and regional differences". Public Health Nutrition, 13(10A), pp. 1688-1692.

Júlíusson, P. B.; Eide, G. E.; Roelants, M.; Waaler, P. E.; Hauspie, R. e BJeRkNes, R. (2010) - "Overweight and obesity in Norwegian children: prevalence and socio-demographic risk factors". Acta Paediatrica, 99(6), pp. 900-905.

Kalabiska, I.; Uvacsek, M.; Petrekanits, M.; Cseprekál, O.; Ihasz, F. e FrenkL, R. (2010) - "Comparison of running performances and prevalence of overweight and obesity in Hungarian and Ukrainian adolescents". Acta Physiologica Hungarica, 97(4), pp. 393-400.

Kautiainen, S.; Rimpelä, A.; Vikat, A. e Virtanen, S. M. (2002) "Secular trends in overweight and obesity among Finnish adolescents in 1977-1999". International Journal of Obesity and Related Metabolic Disorders, 26(4), pp. 544-552.

Krassas, G. E.; Tzotzas, T.; Tsametis, C. e Konstantinidis, T. (2001) - "Prevalence and trends in overweight and obesity among children and adolescentes in Thessaloniki, Greece". Journal of Pediatric Endocrinology and Metabolism, 14(5), pp. 1319-1326.

KRUE, S. e Coolidge, J. (2010) - "The prevalence of overweight and obesity among Danish school children”. Obesity Reviews, 11(7), pp. 489-491.

Lake, A. A.; Mathers, J. C.; Rugg-Gunn, A. J. e Adamson, A. J. (2006) - "Longitudinal changes in food habits between adolescence (11-12 years) and adulthood (32-33 years): the ASH30 study". Journal of Public Health, 28 (1), pp. 10-16.

Landsberg, B.; Plachta-Danielzik, S.; Lange, D.; Johannsen, M.; Seiberl, J. e Müller, M. J. (2010) - "Clustering of lifestyle factors and association with overweight in the adolescents of the Kiel Obesity Prevention Study". Public Health Nutrition, 13 (10A), pp. 1708-1715.

Lasserre, A. M.; Chiolero, A.; Cachat, F.; Paccaud, F. e Bovet, P. (2007) - "Overweight in Swiss children and association with children's and parent's characteristics". Obesity, 15(2), pp. 2912-2919.
Leech, R. M.; McNaughton, S. A. e Timperio, A. (2014) - "The clustering of diet, physical activity and sedentary behavior in children and adolescents: a review". International Journal of Behavioral Nutrition and Physical Activity, 11:4

Ludwig, D. S.; Peterson, K. E. e Gortmaker, S. L. (2001) - "Relation between consumption of sugar-sweetened drinks and childhood obesity: a prospective, observational analysis". The Lancet, 357(9255), pp. 505-508.

Malik, V.; Schulze, M. e Hu, F. (2006) - "Intake of sugar-sweetened beverages and weight gain: a systematic review". The American Journal of Clinical Nutrition, 84(2), pp. 274-288.

Nogueira, H. e Lourenco, A. (2013) - "Ambiente, comportamentos e obesidade na população portuguesa.", In: REMOALDO, P. e Nogueira, H. (coord) - Desigualdades Socioterritoriais e Comportamentos em Saúde. Lisboa, Edições Colibri, pp. 93-114.

Padez, C.; Mourão, P.; Moreira, P. e Rosado, V. (2004) "Prevalence and risk factors for overweight and obesity in Portugusese children." Acta Paediatrica, 94(11), pp. 1550-1557.

Pecin, I.; Samovojska, R.; Heinrich, B.; Zeljkovic-Vrkic, T.; Laganovic, M. e Jelakovic, B. (2013) - "Hypertension, overweight and obesity in adolescents: The Cro-Kop Study". Collegium Antropologicum, 37(3), pp. 761-764.

Phillips, S. M.; Bandini, L. G.; Naumova, E. N.; Cyr, H.; Colclough, S.; Dietz, W. H. e Must, A. (2004) - "Energy-dense snack food intake in adolescence: longitudinal relationship to weight and fatness". Obesity Research, 12(3), pp. 461-472.

Schönbeck, Y.; Talma, H.; van Dommelen, P.; Bakker, B.; BuitendiJk, S. E.; Hirasing, R. A. e van BuURen, S. (2001) - "Increase in prevalence of overweight in Dutch children and adolescents: A comparison of nationwide growth studies in 1980; 1997 e 2009". Plos One, 6(11): E27608.

Telama, R. (2009) - "Tracking of physical activity from childhood to adulthood: a review." Obesity Facts, 2(3), pp. 187-195.

Tremblay, M. S. e Willms, J. D. (2003) - "Is the Canadian childhood obesity epidemic related to physical inactivity?". International Journal of Obesity and Related Metabolic Disorders, 27(9), pp. 1100-1105.

Tutkuviene, J. (2007) - “Body mass index, prevalence of overweight and obesity in Lithuanian children and adolescents, 1985-2002." Collegium Antropologicum, 31(1), pp. 109-121.

Vandewater, E. A.; Shim, M. S. e Caplovitz, A. G. (2004) - "Linking obesity and activity level with children's television and video game use". Journal of Adolescence, 27(1), pp. 71-85. 
Villa, I.; Yngve, A.; Poortvliet, E.; GrJibovski, A.; Livv, K.; SJÖströM, M. e HARRO, M. (2007) - "Dietary intake among under-, normal-, and overweight 9-and 15-year-old Estonian and Swedish schoolchildren". Public Health Nutrition, 10(3), pp. 311-322.

Vlaski, E.; Stavric, K.; Isjanovska, R.; Seckova, L. e Kimovska, M. (2006) - "Overweight hyphothesis in asthma and eczema in young adolescents". Allergology et Immunopathology, 34(5), pp. 199-205.
Welsh, J.; Cogswell, M.; Rogers, S.; Rockett, H.; Mel, Z. e GrummerStRAWN, L. (2005) - "Overweight among low-income preschool children associated with the consumption of sweet drinks: Missouri, 1999-2002". Pediatrics, 115(2), pp. E223-E229.

Whelton, H.; Harrington, J.; Crowley, E.; Kelleher, V.; Cronin, M. e PerRY, I. J. (2007) - "Prevalence of overweight and obesity in the Island of Ireland: results from the North South Survey of Children Height, Weight and Body Mass Index". BioMedCentral Public Health, 7, pp.187. 